\title{
L'historiographie de l'enseignement du français langue étrangère en Allemagne. Un abrégé de son évolution
}

Marcus Reinfried

\section{(2) OpenEdition \\ Journals}

Édition électronique

URL : https://journals.openedition.org/dhfles/3705

DOI : $10.4000 /$ dhfles.3705

ISSN : 2221-4038

Éditeur

Société Internationale pour l'Histoire du Français Langue Étrangère ou Seconde

Édition imprimée

Date de publication : 1 janvier 2013

Pagination : 205-221

ISSN : 0992-7654

\section{Référence électronique}

Marcus Reinfried, «L'historiographie de l'enseignement du français langue étrangère en Allemagne. Un abrégé de son évolution », Documents pour l'histoire du français langue étrangère ou seconde [En ligne], 50 | 2013, mis en ligne le 01 janvier 2017, consulté le 30 mars 2023. URL : http:// journals.openedition.org/dhfles/3705; DOI : https://doi.org/10.4000/dhfles.3705

Ce document a été généré automatiquement le 30 mars 2023.

Tous droits réservés 


\title{
L'historiographie de l'enseignement du français langue étrangère en Allemagne. Un abrégé de son évolution
}

\author{
Marcus Reinfried
}

1 L'historiographie de l'enseignement $d u$ français langue étrangère commence en Allemagne dès la fin du XIX ${ }^{e}$ siècle, c'est-à-dire bien avant la fondation de la SIHFLES. Certaines des premières publications n'ont pas perdu de leur pertinence jusqu'à aujourd'hui ; et il est nécessaire de les intégrer dans un bilan de recherche. Néanmoins, de grandes avancées ont été faites ces quarante dernières années par l'édition de recueils de sources et grâce à la spécialisation de la recherche.

2 La SIHFLES a certes influencé quelques-uns des travaux allemands parus dans les 25 dernières années. Mais le propos de cet article n'est pas de cerner cet apport à peine définissable empiriquement ni de répertorier les articles ayant traité des aspects de l'histoire de l'enseignement du français langue étrangère dans les pays germanophones publiés par la SIHFLES ${ }^{1}$. L'article a été rédigé dans le but d'éclairer le champ de l'historiographie qui forme l'arrière-plan en langue allemande de ce qu'on peut lire dans les publications françaises. Un tel article est nécessairement un abrégé. Il se limite à quelques titres, privilégie surtout les monographies, et ne peut être forcément qu'incomplet. Les titres les plus importants sont répertoriés dans la bibliographie à la fin de l'article. Les autres indications bibliographiques se trouvent dans des notes en bas de page.

\section{Les débuts}

Le Allgemeiner Deutscher Neuphilologen-Verband, la première association allemande des enseignants de langues modernes, a été fondée en 1886. Parmi les fondateurs, il y avait, outre des professeurs de langues, quelques professeurs d'université, des spécialistes en 
philologie romane ou anglaise. Le phonéticien et angliciste Wilhelm Viëtor s'est engagé dans cette association ainsi que son collègue Edmund Stengel, romaniste dans la même université de Marburg. Stengel a établi un célèbre répertoire des grammaires de la langue française jusqu'à la fin du XVIII ${ }^{e}$ siècle, imprimé en 1890 et réédité en 1976 (avec un grand nombre d'ajouts) par Hans-Josef Niederehe. Le romaniste de Marburg a été soutenu dans son travail par environ quarante membres du Allgemeiner Deutscher Neuphilologen-Verband, professeurs des écoles en majorité. Comme il l'explique dans le texte d'une conférence tenue au troisième congrès des néophilologues à Dresde et imprimé à titre d'introduction dans le répertoire (Stengel 1890:1-5), celui-ci était censé être utile non pas seulement à la recherche diachronique de la langue française et de la grammaticographie, mais aussi à une recherche historique approfondie des techniques de l'enseignement du français.

Néanmoins, ce ne sera pas Stengel, bien qu'il se soit intéressé à l'enseignement des langues et ait même adhéré au mouvement réformiste, qui mettra personnellement en œuvre une telle recherche didactique, étant donné le caractère essentiellement linguistique de ses activités et axé sur les lettres. Ce sera Karl Dorfeld, professeur dans un lycée classique à Gießen, qui (peut-être aussi inspiré par Stengel) rédigera le premier texte sur l'histoire de l'enseignement du français en Allemagne (Dorfeld 1892). Il a été imprimé comme appendice au "rapport annuel» de son lycée, destiné à l'information des parents d'élèves ${ }^{2}$. Le texte a ensuite été remanié et complété à plusieurs reprises pour être imprimé dans une grande encyclopédie pédagogique ${ }^{3}$. L'auteur s'intéresse déjà à beaucoup d'aspects différents: il donne de nombreuses informations sur les premières apparitions de l'enseignement $d u$ français dans certaines villes ou écoles allemandes, sur le nombre d'années d'apprentissage et d'heures de cours par semaine, les programmes et les examens. Certaines activités comme l'entraînement oral, les traductions et la rédaction de lettres sont bien décrites. Même les manuels de Meidinger, Seidenstücker, Ahn et Ploetz apparaissent déjà comme les jalons allemands de la méthode grammaire-traduction dans le texte de Dorfeld. La description des méthodes et l'analyse des œuvres littéraires lues dans l'enseignement de niveau avancé sont seulement effleurées. Dans l'emploi des sources, on trouve déjà une assez grande diversification : Dorfeld s'est aussi bien servi de quelques préfaces de vieilles grammaires que de vieux romans dans lesquels les auteurs mentionnent une connaissance des langues et la façon dont les personnes l'acquièrent; il a exploité des sources de l'histoire de la pédagogie (par exemple Monumenta Germaniae Paedagogica) ainsi que de nombreuses descriptions historiques d'écoles allemandes riches de traditions parues à l'occasion de leurs anniversaires dans les Schulprogramme (cf. note 2) ou dans des monographies séparées.

5 Le travail de Dorfeld, assez positiviste et solidement documenté n'a pourtant pas suscité, semble-t-il, beaucoup d'intérêt parmi les enseignants de français engagés dans le mouvement réformiste. Mais au cours de la première décennie du $\mathrm{XX}^{\mathrm{e}}$ siècle, le sentiment se répandit en Allemagne que la «querelle entre les anciens et les modernes » dans l'enseignement des langues étrangères était épuisée et qu'une méthode mixte allait prendre le dessus ${ }^{4}$. Les controverses entre les propagateurs de la méthode directe et ceux de la méthode grammaire-traduction s'apaisèrent et certains spécialistes de l'enseignement des langues étrangères commencèrent à s'intéresser à l'histoire pour pouvoir mieux classer et comprendre les alternatives méthodiques du présent. Pratiquement tous les articles historiques parus pendant ces années-là qui 
prenaient la relève de Dorfeld ${ }^{5}$ mettaient par conséquent surtout l'accent sur les méthodes de l'enseignement des langues. On intégra aussi des chapitres historiques dans certains ouvrages de référence destinés à la formation pratique des professeurs ${ }^{6}$.

Albert Streuber (1914) est l'auteur de la première monographie traitant de l'histoire des méthodes du XVI ${ }^{\mathrm{e}}$ au XVIII ${ }^{\mathrm{e}}$ siècle, thèse soutenue à l'université de Gießen. Streuber fondait sa recherche surtout sur les grammaires répertoriées par Stengel (1890). Son intention était de corriger les travaux de Dorfeld et de Lehmann ( $c f$. notes 3 et 5) qui lui semblaient trop schématiques et lacunaires dans le champ très vaste de l'histoire des grammaires françaises utilisées en Allemagne ${ }^{7}$ (Streuber 1914 : 9-11). Aussi a-t-il décrit plus de 200 grammaires dans sa thèse. Mais l'utilité de ce travail appliqué est très limitée car Streuber y classe ses auteurs de grammaires (en général aussi des maitres de la langue française) essentiellement selon trois catégories : adhérents à une méthode déductive, à une méthode inductive ou à une méthode mixte. Or ces termes restent flous et inexpliqués tout au long de son texte; ils ne sont définis nulle part. On peut supposer que pour Streuber la méthode déductive n'était pas seulement caractérisée par la primauté des règles grammaticales, mais aussi par la primauté des compétences écrites, tandis que la méthode inductive appréciait surtout la conversation et trouvait son point de départ dans un texte ou dans une phrase. Mais Streuber ne distinguait pas non plus entre les méthodes qui incitaient les apprenants à trouver par eux-mêmes certaines règles grammaticales et les méthodes qui renonçaient largement à la conscientisation. En outre l'indication des références par Streuber est incomplète : il ne désigne souvent ses grammaires que par le nom de leur auteur, il n'en donne souvent ni les titres ni les années de parution ni les pages dans ses citations ${ }^{8}$. Néanmoins, il reste jusqu'à présent le seul historien en Allemagne qui ait essayé de réaliser (bien que de façon peu fiable et contrôlable) la description des techniques de l'enseignement du français proposée par Stengel $(1890: 2)$.

7 Vers 1910, l'approche scientifique dominante dans les sciences humaines commence à se transformer en Allemagne. Les dernières décennies avaient surtout été influencées par le paradigme empiriste, les années vingt seront plutôt placées sous un renouveau de la pensée idéaliste. L'histoire des idées gagna en importance. L'étude de Hugo Dietze (1927), la première analyse diachronique de l'apprentissage des langues étrangères dans la formation professionnelle des commerçants allemands, est par son sujet nécessairement liée à l'histoire économique. Mais l'abrégé du développement de l'enseignement des langues modernes rédigé par Walter Hübner ${ }^{9}$ (1929), inspecteur dans l'administration scolaire en Prusse et professeur honoraire à la FriedrichWilhelm-Universität à Berlin, est surtout orienté vers l'évolution des fonctions éducatives des langues française et anglaise. La thèse de Bernhard Schmidt (1931), soutenue à l'Institut des Sciences de l'éducation de l'université de Halle, décrit l'enseignement de la langue française au XVII ${ }^{e}$ siècle surtout dans le contexte d'idées pédagogiques générales (comme celles exprimées par Ratke et par Comenius), même s'il a aussi analysé assez précisément environ 90 manuels de français parus à cette époque.

\section{Les recueils de sources}

8 Entre le début du Troisième Reich et le milieu des années cinquante, la recherche historique concernant l'enseignement des langues étrangères s'est arrêtée en 
Allemagne. Les nazis ont en général accordé peu d'importance à l'enseignement des langues, surtout au français qui était considéré comme la langue d'un «ennemi héréditaire " et associé à une culture et une race considérées comme décadentes. Le français, traditionnellement la langue étrangère moderne la plus importante en Allemagne, fut dégradé à partir de 1938 et devint une matière optionnelle dans la plupart des lycées avec peu d'heures d'enseignement (Reinfried 2003 [2001] : 130-133, 137-138). Pendant la Deuxième Guerre mondiale et pendant les années de la reconstruction de l'Allemagne, l'enseignement des langues étrangères a été peu pris en considération par la recherche universitaire.

9 La recherche reprend en RDA grâce à Koch $^{10}$ qui étudie les statuts professionnels, les activités et les conditions de vie des maîtres de langues étrangères du XVII ${ }^{e}$ au XIX ${ }^{e}$ siècle (avec les débuts de la romanistique) à l'université de Iéna. En RFA, Flechsig traite dans sa thèse (1962), soutenue à l'Institut de Pédagogie de l'université de Göttingen ${ }^{11}$, des conceptions éducatives dépendant entre autres des conceptions du culturel et des conceptions du langage, dans l'histoire de l'enseignement des langues étrangères modernes. Même si cette thèse, qui n'a jamais été imprimée, donne à la lecture parfois l'impression d'une première version d'un texte hâtivement écrit avec certaines faiblesses quant au contenu ( $c f$. Schröder 1990 : 4), elle a inspiré à l'auteur la publication de deux volumes (Flechsig 1965, 1970) contenant des préfaces de manuels et des articles qui illustrent bien l'évolution historique des attentes éducatives liées à l'enseignement des langues.

Konrad Schröder (1969) décrit dans sa thèse le développement de l'enseignement de l'anglais dans les universités germanophones ${ }^{12}$. Cette thèse est composée pour moitié d'appendices classant par ordre alphabétique les universités offrant des cours d'anglais ou en langue anglaise ainsi qu'une liste alphabétique de lecteurs et professeurs enseignant cette langue. Ce projet de rassembler systématiquement des données concernant l'histoire de l'enseignement des langues étrangères est poursuivi par Schröder dans les décennies suivant sa thèse ; il devient l'éditeur de recueils de sources le plus important en Allemagne, probablement même en Europe, dans ce domaine. Schröder commence avec les Linguarum recentium annales (1980, 1982, 1983, 1985), environ 3000 petits extraits de littérature secondaire allemande arrangés en ordre chronologique qui contiennent des informations concernant la parution de grammaires et d'autres manuels, l'emploi de maîtres de langues, les conditions de l'enseignement des langues dans des institutions scolaires ainsi que des descriptions de l'usage de langues dont on peut déduire l'importance sociale dans certains milieux. Ces " annales" se limitent à la période entre 1500 et 1800 . Elles contiennent beaucoup d'informations sur l'enseignement du français qui a été pendant toute cette époque la langue étrangère dominante en Allemagne (Schröder 1980 : XIII), mais aussi sur celui de l'italien, de l'anglais et de l'espagnol et même sur les « petites langues étrangères » - du point de vue de leur diffusion en Allemagne -, comme le portugais, le hongrois ou l'arabe.

11 Le deuxième grand recueil est un dictionnaire biographique (en six volumes) des enseignants de langues étrangères dans les pays germanophones avant 1800 . Schröder y a rassemblé environ 3800 articles $^{13}$. Il ajoute dans des bibliographies à la fin des volumes environ 5000 titres de monographies ou d'articles, en majorité des études d'histoire locale ou régionale, dans lesquelles il a trouvé des données biographiques. Mais les indications bibliographiques, classées surtout selon des critères 
géographiques, ne peuvent pas être facilement mises en rapport avec des articles précis, et dans les articles, les références manquent - même pour les citations. Espérons qu'il y aura une deuxième édition de ce répertoire, annoncée déjà par Schröder dans un entretien personnel, avec les références nécessaires dans chaque article qui permettront de vérifier l'exactitude des données.

12 Nous devons aussi plusieurs recueils de sources à Herbert Christ. Un petit recueil de Christ \& Liebe (1979 [1978]) est consacré à la politique des langues scolaires en RFA ${ }^{14}$. Un deuxième, paru à la Wissenschaft-liche Buchgesellschaft qui publie surtout des livres universitaires en sciences humaines, est censé représenter une anthologie du développement de la pensée didactique de l'enseignement du français. Il comprend une trentaine de textes, parus pour la première fois entre 1613 et 1978. Le troisième recueil, édité par Christ \& Rang (1985), réunit en 7 volumes environ 600 programmes scolaires et arrêtés concernant les examens ou les horaires dans l'enseignement des langues étrangères en Allemagne. Ces documents couvrent la période de 1700 à 1945. Ils sont utiles pour analyser les objectifs d'enseignement, c'est-à-dire les compétences dans l'emploi de la langue cible ainsi que les connais-sances culturelles et grammaticales qu'on souhaitait faire acquérir aux élèves. Certains programmes donnent aussi des renseignements méthodiques ou des informations sur le choix des œuvres littéraires à lire dans les dernières années de la scolarité secondaire.

\section{La recherche spécialisée}

Dans le dernier tiers du $\mathrm{XX}^{\mathrm{e}}$ siècle, parallèlement aux progrès dans la collecte et le classement des sources, la publication de la littérature secondaire a gagné en intensité. Plusieurs travaux décrivent le développement historique des contenus dans l'enseignement du français langue étrangère. Walter Apelt (1967) analyse dans son mémoire de qualification pour l'enseignement supérieur en RDA la Kulturkunde, c'est-àdire la doctrine du caractère des peuples qui a beaucoup influencé l'enseignement des langues étrangères en Allemagne dans les années 1920 et qui a été combinée avec une pseudo-science des races pendant la période du Troisième Reich. Ce livre, méritoire dans les années soixante parce qu'il thématisait une évolution passée longtemps sous silence en RFA, ne distingue pourtant pas suffisamment, malgré l'utilisation d'un très grand nombre de sources, les différentes manifestations du mouvement de la Kulturkunde. Un long article de Reinfried (1999) essaie de mieux différencier, spécialement pour l'enseignement du français, plusieurs phases du développement de la Kulturkunde et décrit les déterminants qui ont influencé son évolution.

On remarquera également les trois thèses publiées par Hans Bode (1980), Jürgen Krauskopf (1985) et Helmut Niederländer (1981) qui s'intéressent aussi au développement historique des contenus dans l'enseignement du français langue étrangère en analysant des manuels de français. Hans Bode analyse les manuels d'August Grund, très répandus en Allemagne entre 1913 et 1969 et parus dans de nombreuses versions et éditions différentes. Il s'intéresse surtout aux sujets traités dans les textes, à la structure de l'enseignement de la civilisation française et à l'évolution de l'image de la France en Allemagne. Jürgen Krauskopf analyse l'évolution des stéréotypes dans 23 manuels de français parus en Allemagne et 25 manuels d'allemand parus en France entre 1950 et 1980. Ces images de l'autre se rapportent aux centres d'intérêt suivants : famille, école, loisirs, régions, histoire, politique, littérature. 
Quant à Helmut Niederländer, il analyse 17 grammaires de la langue française imprimées en Allemagne entre 1850 et 1950. Sur la base de ce corpus, il établit une typologie des grammaires, en définit les différentes structurations ainsi que les contenus et décrit les traits spécifiques des grammaires didactiques.

En ce qui concerne l'analyse des méthodologies et méthodes, il n'y a pas encore eu d'ouvrages de référence, publiés dans le domaine de l'histoire de l'enseignement du français en Allemagne; les monographies parues se limitent à l'enseignement de l'anglais. Mais certains résultats des recherches liées à ces publications devraient néanmoins être en partie applicables à l'enseignement du français. Konrad Macht $(1986,1987,1990)$ se sert essentiellement d'extraits de manuels pour la description de ses treize méthodologies des $\mathrm{XIX}^{\mathrm{e}}$ et $\mathrm{XX}^{\mathrm{e}}$ siècles. Il utilise une grille d'analyse qui évalue pour chaque méthodologie l'importance relative des quatre aptitudes langagières (écouter, parler, lire et écrire), de la connaissance d' " unités langagières » (lexique, structures grammaticales) et de l'intégration de différents domaines thématiques concernant la culture cible. Friederike Klippel (1994 : 22-27) en revanche n'accepte pas sa notion de méthode qui, selon elle, est trop restreinte. Elle se base sur la modélisation de Richards \& Rodgers ${ }^{15}$ (1986 : 14-30) distinguant entre trois niveaux descriptifs d'une méthode: le niveau théorique (approach), le niveau de la complexion des facteurs d'enseignement/apprentissage (design) et le niveau des techniques d'enseignement/ apprentissage (procedure). Klippel (1994: 16-17) examine surtout le niveau moyen du design en analysant un corpus d'environ 300 manuels (parus aux XVIII ${ }^{\mathrm{e}}$ et XIX ${ }^{\mathrm{e}}$ siècles) ainsi que les réflexions méthodiques correspondantes.

Quant à la question des supports dans l'enseignement des langues étrangères d'un point de vue historique, elle est traitée d'une part par Hanno Schilder (1977) et d'autre part par Marcus Reinfried (1992) dans leurs thèses de doctorat respectives, publiées sous forme de monographies. Schilder décrit la " méthode par l'aspect » (qui utilise des objets et des tableaux muraux) et la « méthode auditive » (qui intègre des disques et des émissions de radio dans l'enseignement des langues modernes) entre 1880 et 1945 . Il analyse surtout un grand nombre d'articles de revues. Reinfried se limite à l'emploi des images dans l'enseignement du français, mais il traite ce sujet du début du XVI ${ }^{\mathrm{e}}$ siècle jusqu'à l'année 1990. Il s'intéresse surtout à la question de savoir s'il y a, à certaines époques, des parallèles et influences réciproques entre un "sensualisme didactique ", s'exprimant dans l'emploi d'images, de modèles réduits, d'une pédagogie "naturelle » et de la méthode directe, et une tendance épistémologique vers le sensualisme dans les humanités. Il a aussi déduit de son analyse historique une typologie des fonctions didactiques de l'image (Reinfried 1992 : 245-258).

La politique des langues qui forme le contexte de l'enseignement de la langue française et d'autres langues étrangères en Allemagne a été traitée dans une monographie de Herbert Christ $^{16}$ qui intègre aussi un certain nombre de réflexions historiques, même si le XIX ${ }^{\mathrm{e}}$ siècle et les siècles antérieurs y sont peu pris en considération. De plus, Christ aussi bien que l'angliciste Schröder, qui n'a néanmoins pas écarté l'enseignement du français dans ses travaux ${ }^{17}$, se sont beaucoup intéressés à l'histoire sociale de l'enseignement des langues. Cela ne se manifeste pas seulement dans leurs articles, mais aussi d'une façon nette dans les recueils de sources qu'ils ont publiés (voir cidessus). Pour certains aspects du «contexte systémique» de l'enseignement des langues ${ }^{18}$, on trouve fréquemment dans les projets de recherche en cours ou rédigés ces dernières années (surtout les thèses des anglicistes ou des romanistes) des approches 
combinant l'histoire sociale avec l'histoire culturelle. Christiane Obermeier (2012), pour donner un exemple, décrit l'implantation des langues modernes en Prusse entre 1859 et $1931 \mathrm{du}$ point de vue des institutions scolaires et de la perspective sociale des apprenants et de leurs parents, mais elle prend aussi en compte le point de vue de l'histoire culturelle en analysant les débats qui avaient lieu entre les défenseurs des langues classiques et ceux des langues modernes au XIX siècle ainsi qu'entre les partisans du français et les propagateurs de l'anglais dans les années vingt du $\mathrm{XX}^{\mathrm{e}}$ siècle. Les quelques historiens de l'enseignement des langues étrangères présents dans les universités allemandes sont souvent confrontés aux nombreux didacticiens, fervents adeptes de la méthodologie empirique des sciences sociales et méprisant parfois l'histoire, et sont donc parfois contraints d'expliquer et de justifier d'une manière offensive leur approche scientifique; la diachronie, elle aussi, a besoin d'une réflexion méthodologique et d'une théorisation spécifique. On commence fort heureusement à intégrer la méthodologie de la recherche historique des langues étrangères dans les manuels de recherche empirique ${ }^{19}$.

Les acteurs de l'enseignement du français, les enseignants et les apprenants, n'ont pas encore été analysés de manière systématique. Christ (1983) a décrit les différents types de maitres de langue ainsi que les professeurs modernes et leur formation dans plusieurs articles, partiellement avec quelques exemples, mais sans se baser sur un corpus avec des indications biographiques. Doff (2002: 247-339) a rédigé dans sa thèse sur l'enseignement des langues modernes aux jeunes filles dans la deuxième moitié du $\mathrm{XIX}^{\mathrm{e}}$ siècle de longs passages décrivant l'institutionnalisation d'écoles supérieures réservées aux filles comprenant la formation des enseignantes et leurs conditions matérielles. En ce qui concerne les apprenants de français, nous ne connaissons ni leur nombre à différentes époques ni leur répartition dans les différentes régions allemandes, ni leurs appartenances à telle ou telle classe sociale. Ce serait déjà un progrès considérable si on arrivait à avoir des chiffres approximatifs pour les XIX ${ }^{\mathrm{e}}$ et $\mathrm{XX}^{\mathrm{e}}$ siècles. Marcus Reinfried (2012) a calculé les pourcentages annuels dans les Realschulen et les lycées allemands en commençant par la période entre 1965 et $2012^{20}$ une tâche ô combien compliquée dans un pays fédéral aussi divisé que l'Allemagne si on continue avec la période entre 1815 et 1933.

\section{Vers une représentation d'ensemble}

19 Même si on est encore loin d'avoir étudié de façon approfondie tous les aspects et toutes les époques de l'enseignement du français langue étrangère en Allemagne, on ne peut pas nier les avancées des dernières décennies. Un grand nombre d'articles et de monographies spécialisés ont été publiés. L'histoire de l'enseignement du français est bel et bien présente (en général avec les autres langues modernes) dans les grands manuels et dictionnaires de la didactique des langues ou de la franco-romanistique ${ }^{21}$. Néanmoins, les progrès de la recherche des dernières décennies sont dans l'ensemble encore présentés de manière trop éparpillée. Il n'existe pas encore de monographies présentant une vue d'ensemble de l'évolution de l'enseignement/apprentissage du français langue étrangère en Allemagne.

20 Il existe pourtant une "Petite histoire de l'enseignement des langues étrangères " rédigée en langue allemande par un angliciste (Hüllen 2005). Mais cette vue d'ensemble, traitant de l'évolution historique depuis l'antiquité jusqu'à nos jours en 150 pages 
environ, ne décrit précisément qu'une partie des aspects pertinents et en effleure seulement d'autres, non moins importants. Les conceptions éducatives y sont par exemple bien décrites, mais les conceptions du culturel et surtout les méthodes restent relativement floues, les enseignants et les apprenants sont insuffisamment caractérisés. Et le français langue étrangère dans ses particularités parmi les autres langues étrangères n'est pas assez pris en compte. Mais Walter Kuhfuß, membre de la SIHFLES, qu'on connaissait jusqu'à présent seulement comme spécialiste des débuts de l'enseignement du français en Allemagne au XVI ${ }^{e}$ siècle ${ }^{22}$ comblera en partie cette lacune. Il vient de terminer un long texte d'environ 740 pages, une histoire culturelle de l'enseignement du français de 1490 à 1815 en Allemagne, qui n'écarte pas l'histoire sociale. Le livre (Kuhfuß 2013) paraitra à la fin de l'année 2013.

\section{BIBLIOGRAPHIE}

APELT, Walter (1967). Die kulturkundliche Bewegung im Unterricht der neueren Sprachen in Deutschland in den Jahren 1886 bis 1945. Berlin (Est) : Verlag Volk und Wissen.

BODE, Hans (1980). Die Textinhalte in den Grundschen Französischlehrbüchern. Eine fachdidaktische Untersuchung von Lesestoffen französischer Sprachlehrbücher für höhere Schulen in Deutschland zwischen 1913 und 1969. Europäische Hochschuls-chriften, Série XI, Vol. 90. Frankfurt a. M. : Lang.

CHRIST, Herbert (1983). « Zur Geschichte des Französischunterrichts und der Französischlehrer » In Anneliese Mannzmann (dir.). Geschichte der Unterrichtsfächer I. München : Kösel, 95-117.

CHRIST, Herbert \& RANG, Hans-Joachim (éd.) (1985). Fremdsprachenunterricht unter staatlicher Verwaltung. Eine Dokumentation amtlicher Richtlinien und Verordnungen. Vol. I : Einleitung und Orientierung. Vol. II : Allgemeine Anweisungen für den Fremdsprachenunterricht. Vol. III : Neuere Fremdsprachen I. Vol. IV : Fremdsprachen II. Vol. V : Alte Sprachen. Vol. VI : Prüfungsbestimmungen für den Fremdsprachenunterricht. Vol. VII : Der Fremdsprachenunterricht in Stundentafeln. Tübingen : Narr.

DIETZE, Hugo (1927). « Geschichte der fremdsprachlichen Ausbildung des Kaufmanns » In id. Methodik des fremdsprachlichen Unterrichts an Handelsschulen. Handbuch für das kaufmännische Unterrichts-wesen in Deutschland, tome 2, 6 ${ }^{\mathrm{e}}$ partie. Leipzig : Gloeckner, 7-56.

DOFF, Sabine (2002). Englischlernen zwischen Tradition undInnovation. Fremdsprachenunterricht für Mädchen im 19.Jahr-hundert. München : Langenscheidt-Longman.

DORFELD, Karl (1892). Beiträge zur Geschichte des französischen Unterrichts in Deutschland. Beilage zum Programm des Großherzoglichen Gymnasiums in Gießen 1891/92. Gießen : Keller.

FLECHSIG, Karl-Heinz (1962). Die Entwicklung des Verständnisses der neusprachlichen Bildung in Deutschland. Thèse de doctorat. Université de Göttingen. (Manuscrit.)

FLECHSIG, Karl-Heinz (dir.) $(1965,1970)$. Neusprachlicher Unterricht, I et II. Quellen zur Unterrichtslehre, 10 et 11 . Weinheim : Beltz.

HÜLLEN, Werner (2005). Kleine Geschichte des Fremdsprachen-lernens. Berlin : Schmidt. 
KLIPPEL, Friederike (1994). Englischlernen im 18. und 19. Jahrhundert. Die Geschichte der Lehrbücher und Unterrichts-methoden. Münster : Nodus.

KRAUSKOPF, Jürgen (1985). Das Deutschland- und Frankreichbild in Schulbüchern. Deutsche Französischbücher und französische Deutschbücher von 1950 - 1980. Tübingen : Narr.

KUHFUSS, Walter (à paraître). Histoire culturelle de l'enseignement du français en Allemagne. 1490 1815 [titre provisoire]. Environ 600 p.

KUHFUSS, Walter (2013, à paraître). Eine Kulturgeschichte des Französischunterrichts in der frühen Neuzeit (1490-1815) in Deutschland. Französischlernen am Fürstenhof, auf dem Marktplatz und in der Schule. Göttingen: V\&R unipress.

MACHT, Konrad (1986, 1987, 1990). Methodengeschichte des Englischunterrichts. Vol. 1 : 1800-1880. Vol. 2 : 1880-1960. Vol. 3 : 1960-1983. Augsburger I- \& I-Schriften, 35, 39, 53. Augsburg : Universität.

NIEDERLÄNDER, Helmut (1981). Französische Schulgrammatiken und schulgrammatisches Denken in Deutschland von 1850 bis 1950. Ein Beitrag zur Lehrwerkanalyse. Europäische Hochschulschriften, série XIII, vol. 70. Frankfurt a. M. : Lang.

OSTERMEIER, Christiane (2012). Die Sprachenfolge an den höheren Schulen in Preußen. Ein historischer Diskurs. Stuttgart : ibidem.

REINFRIED, Marcus (1992). Das Bild im Fremdsprachenunterricht. Eine Geschichte der visuellen Medien am Beispiel des Französisch-unterrichts. Tübingen : Narr.

REINFRIED, Marcus (1999). « Von der Realien- zur Kulturkunde. Frankreichkundliche Paradigmen als dialogische Konstrukte im deutschen Französischunterricht » In Herwart Kemper, Siegfried Protz \& Detlef Zöllner (dir.). Schule - Bildung - Wissenschaft. Dia-Logik in der Vielfalt. Rudolstadt/ Jena : Hain, 199-234.

SCHILDER, Hanno (1977). Medien im neusprachlichen Unterricht seit 1880. Eine Grundlegung der Anschauungsmethode und der auditiven Methode unter entwicklungsgeschichtlichem Aspekt.

Monographien Fremdsprachen - Theorie und Praxis ihrer Didaktik, 2. Kronberg / Taunus : Scriptor.

SCHMIDT, Bernhard (1931). Der französische Unterricht und seine Stellung in der Pädagogik des 17. Jahrhunderts. Hallische Pädagogische Studien, 13. Halle an der Saale : Klinz.

SCHRÖDER, Konrad (éd.) $(1980,1982,1983,1985)$. Linguarum recentium annales. Der Unterricht in den modernen europäischen Sprachen im deutschsprachigen Raum. Vol. 1 : 1500-1700. Vol. 2 : 1701-1740. Vol. 3 : 1741-1770. Vol. 4 : 1771-1800. Augsburger I- \& I-Schriften, 10, 18, 23, 33. Augsburg : Universität.

SCHRÖDER, Konrad (1990). «L'enseignement des langues étrangères en Allemagne. Motifs, centres d'intérêt, résultat et portée ». Documents pour l'histoire du français langue étrangère ou seconde, 5, 2-9.

SCHRÖDER, Konrad (éd.) (21991, 1989, 1992, 1995, 1996, 1999). Biographisches und bibliographisches Lexikon der Fremdsprachen-lehrer des deutschsprachigen Raumes, Spätmittelalter bis 1800. Vol. 1 : Buchstaben A bis C - Quellenverzeichnis. Vol. 2 : Buchstaben D bis H - Erste Nachträge zum Quellenverzeichnis. Vol. 3 : Buchstaben I bis Q. Vol. 4 : Buchstaben R bis Z - Zweite Nachträge zum Quellenverzeichnis. Vol. 5 : Nachträge und Ergänzungen Buchstaben A bis K. Vol. 6 : Nachträge und Ergänzungen Buchstaben L bis Z - Dritte Nachträge zum Quellenverzeichnis. Augsburger I- \& I-Schriften, 40, 51, 63, 68, 73, 74. Augsburg : Universität.

STENGEL, Edmund (1976 [1890]). Chronologisches Verzeichnis französischer Grammatiken vom Ende des 14. bis zum Ausgange des 18. Jahrhunderts nebst Angabe der bisher ermittelten Fundorte derselben. Éd. 
par Hans-Joachim Niederehe avec un appendice. Amsterdam Studies in the theory and history of linguistic science, série 3, vol. 8. Amsterdam : Benjamins.

STREUBER, Albert (1914). Beiträge zur Geschichte des französischen Unterrichts im 16. bis 18.

Jahrhundert. Teil I : Die Entwicklung der Methoden im allgemeinen und das Ziel der Konversation im besonderen. Romanische Studien, 15. Berlin : Ebering.

\section{NOTES}

1. Les articles parus dans Documents pour l'histoire du français langue étrangère ou seconde, rédigés par des Allemands et cités dans Marie-Christine Kok Escalle, Nadia Minerva \& Marcus Reinfried (coord.) (2012). Histoire internationale de l'enseignement du français langue étrangère ou seconde: problèmes, bilans et perspectives. Recherches et applications. Le français dans le monde, 52, y sont répertoriés aux pages 160-168; ils offrent un résumé de la recherche menée pendant les 25 ans d'existence de la SIHFLES. Il s'agit de contributions de Herbert Christ, Henning Düwell, Gerda Hassler, Erika Hültenschmidt, Konrad Macht, Esther Möller, Marcus Reinfried, Konrad Schröder et Franz-Rudolf Weller.

2. Ces "Schulprogramme", institutionnalisés dans les lycées et collèges, contenaient des informations sur les matières, les contenus et les examens, les noms des enseignants et des élèves dans les classes, éventuellement des descriptions de fêtes scolaires avec des discours tenus à cette occasion. À partir du début du XIX siècle, ils ont été complétés par un ou deux articles scientifiques ou pédagogiques (d'une vingtaine de pages) à travers lesquels les professeurs faisaient la preuve de leurs connaissances.

3. Karl Dorfeld (1896). «Französischer Unterricht, geschichtlicher Abriß». In Wilhelm Rein (dir.). Encyclopädisches Handbuch der Pädagogik, vol. 2. Langen-salza : Beyer, 395-419; id. (1905). « Französischer Unterricht, geschichtlicher Abriß» In Wilhelm Rein (dir.), op. cit., 1-31.

4. Georg Steinmüller (1909). Hermann Breymann's Neusprachliche Reform-Literatur. Eine bibliographisch-kritische Übersicht. Vol. 4: 1904-1909. Leipzig : Deichert, 151.

5. Wilhelm Viëtor (1902). Die Methodik des neusprachlichen Unterrichts. Ein geschichtlicher Überblick in vier Vorträgen. Sammlung neuphilologischer Vorträge und Abhandlungen, 3. Leipzig : Teubner ; Alwin Lehmann (1904). "Der neusprachliche Unterricht im 17. und 18. Jahrhundert, insbesondere seine Methode im Lichte der Reform der Neuzeit ». In Jahresbericht der Annenschule (Real-gymnasium) zu Dresden-Altstadt, Dresden : Teubner, 3-40 ; Gerhard Budde (1906). «Ein Gang durch Jahrhunderte sprachlicher Methodik » Neue Jahrbücher für das klassische Altertum, Geschichte und deutsche Literatur und für Pädagogik, 18, 263-271; Otto Boerner \& Ernst Stiehler (1906). «Zur Geschichte der neueren Sprachen ». Neue Jahrbücher für das klassische Altertum, Geschichte und deutsche Literatur und für Pädagogik, 18, 334-351, 392-412, 459-471.

6. Otto Wendt ( ${ }^{3} 1909$ [11888]). «Methodischer Betrieb der französischen Sprache "In id. Enzyklopädie des französischen Unterrichts, Methodik und Hilfsmittel für Studierende und Lehrer der französischen Sprache mit Rücksicht auf die Anforderungen der Praxis. Hannover/Berlin: Meyer, 36-132 ; Benno Röttgers (1913). « Der Unterricht im Französischen. I. Geschichtliches » In id. Methodik des französischen und englischen Unterrichts in höheren Lehranstalten jeder Art. Methodik des elementaren und höheren Schulunterrichts. Für den Gebrauch in Seminaren und Oberlyzeen, 3, 1-26.

7. Stengel (1890) lui-même a déjà répertorié 625 grammaires dont presque la moitié a connu plusieurs éditions, se distinguant quelquefois considérablement les unes des autres.

8. Streuber (1914:8) exclut même les indications nécessaires à une identification précise dans sa bibliographie, et renvoie pour cela globalement au répertoire de Stengel. 
9. Walter Hübner (1933 [1929]). Didaktik der neueren Sprachen. Handbuch des Unterrichts an höheren Schulen. Zur Einführung und Weiterbildung in Einzeldarstellungen. Frankfurt a. M. : Diesterweg, 1-21.

10. Herbert Koch (1955). Geschichte der Romanistik an der Universität Jena, IV + 126 p. (Manuscrit dans les archives de l'université de Iéna, cote H/C 155, avec un index des noms de personnes et un appendice des annotations, cote H8C 155/2).

11. Karl-Heinz Flechsig (1962). Die Entwicklung des Verständnisses der neusprachlichen Bildung in Deutschland. Thèse de doctorat. Manuscrit à l'université de Göttingen.

12. Konrad Schröder (1969). Die Entwicklung des englischen Unterrichts an deutschsprachigen Universitäten bis zum Jahre 1850. Mit einer Analyse zu Verbreitung und Stellung des Englischen als Schulfach an den deutschen höheren Schulen im Zeitalter des Neuhumanismus. Ratingen : Henn.

13. Quelques-uns de ces articles sont assez longs et contiennent beaucoup de détails, d'autres courts, se limitant dans certains cas même à indiquer un nom, une année et une institution ou une ville dans laquelle une langue étrangère a été enseignée.

14. Herbert Christ \& Elisabeth Liebe (éd.) $\left({ }^{1} 1978,{ }^{2} 1979\right)$. Dokumente zur Schulsprachenpolitik in der Bundesrepublik Deutschland. Augsburger I- \& I-Schriften, 7. Augsburg : Universität.

15. Jack C. Richards \& Theodore S. Rodgers (1986). Approaches and Methods in Language Teaching. A description and analysis. Cambridge : Cambridge University Press, 14-30.

16. Herbert Christ (1980). Fremdsprachenunterricht und Sprachenpolitik. Stuttgart : Klett-Cotta.

17. Par exemple Konrad Schröder (1995). « Französischunterricht in Berlin im 18. Jahrhundert » In Herbert Christ \& Gerda Haßler (dir.). Regards sur l'histoire de l'enseignement des langues étrangères. Actes de la Section 8 du Romanistentag de Potsdam du 27 au 30 septembre 1993. Tübingen : Narr, 188-210.

18. Nadia Minerva \& Marcus Reinfried (2012). «Les domaines à explorer et l'évolution historique ». In Marie-Christine Kok Escalle, Nadia Minerva \& Marcus Reinfried (coord.), op. cit., 20-26.

19. Sabine Doff \& Tim Giesler (2002). « Historische Fremdsprachen-forschung » In Sabine Doff (dir.). Fremdsprachenunterricht empirisch erforschen: Grundlagen - Methoden - Anwendung. Tübingen : Narr, 82-112.

20. Marcus Reinfried (2012). «Der Französischunterricht und seine quantitative Entwicklung an den deutschen Schulen (1965-2012) ». Französisch heute, 43 (4), 178-189.

21. Cf. Reiner Lehberger ( ${ }^{5} 2007$ [ $\left.\left.{ }^{4} 2003\right]\right)$. "Geschichte des Fremdsprachen-unterrichts in deutschsprachigen Ländern bis 1945 ». In Karl-Richard Bausch, Herbert Christ \& Hans-Jürgen Krumm (dir.). Handbuch Fremdsprachenunterricht. Tübingen/Basel : Francke, 609-614 ; Herbert Christ \& Rudolf De Cillia ( ${ }^{5} 2007$ [ $\left.\left.{ }^{4} 2003\right]\right)$. «Geschichte des Fremdsprachen-unterrichts in deutschsprachigen Ländern seit 1945 » In Karl-Richard Bausch, Herbert Christ \& Hans-Jürgen Krumm (dir.). Handbuch Fremdsprachenunterricht. Tübingen/Basel: Francke, 614-621; Konrad Schröder (2010). « Geschichte des Fremdsprachenunterrichts ». In Carola Surkamp (dir.). Metzler Lexikon Fremdsprachendidaktik. Ansätze - Methoden - Grundbegriffe. Stuttgart/Weimar: Metzler/ Poeschel, 87-91; Marcus Reinfried (2008 [12002]). «Der Unterricht des Französischen in Deutschland». In Ingo Kolboom, Thomas Kotschi \& Edward Reichel (dir.). Handbuch Französisch. Sprache, Literatur, Kultur, Gesellschaft. Für Studium, Lehre, Praxis. Berlin : Schmidt, 148-159.

22. Walter Kuhfuß (1976). «Frühformen des Französischunterrichts in Deutschland. Beiträge zur ersten Ausweitungsphase organisierter französischer Sprachunterweisung (1554-1618)». In Harald Haarmann \& Anna Liisa Värri-Haarmann (dir.). Sprachen und Staaten. Festschrift Heinz Kloss. Vol. I: Der politische und soziale Status der Sprachen in den Staaten der Europäischen Gemeinschaft. Schriftenreihe zur europäischen Integration, 15. Hamburg: Stiftung Europa-Kolleg, 323-348 ; id. (2011). «Wie und warum lernte Friedrich der Weise Französisch ? Überlegungen zu den Anfängen des deutschen Französisch-unterrichts. » Französisch heute, 42 (2), 57-64. 


\section{AUTEUR}

\section{MARCUS REINFRIED}

Université Friedrich-Schiller, Iéna 